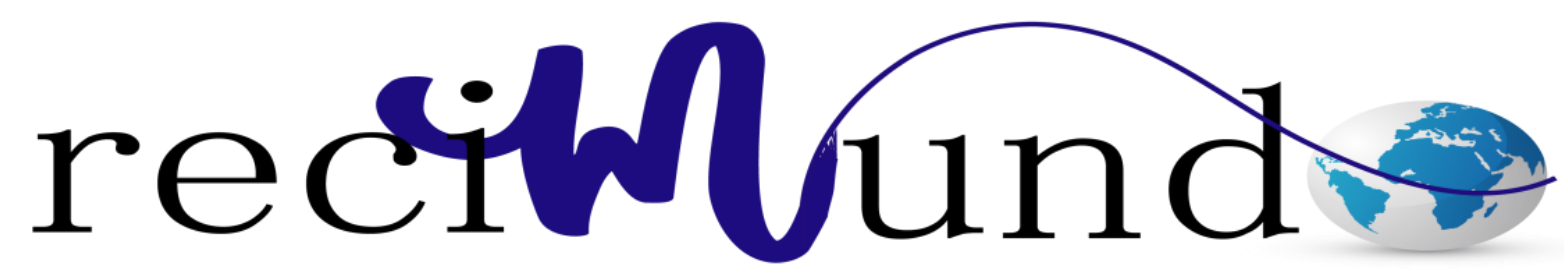

Revista Cientifica Mundo de la Investigación y el Conocimiento

Félix Adolfo Navia Mayorga ${ }^{\text {a. }}$ David Mayorga Arias ${ }^{\text {b; }}$ Ida Ivete Campi Mayorga ${ }^{\text {c; }}$ Luis Alberto De Lucas Coloma ${ }^{\mathrm{d}}$

Liderazgo: una habilidad gerencial fundamental en el éxito de una empresa en el siglo XXI

Leadership: a fundamental managerial skill in the success of a company in the 21 st century

Revista Científica Mundo de la Investigación y el Conocimiento. Vol. 3 núm.3, septiembre, ISSN: 2588-073X, 2019, pp. 1061-1084

DOI: $10.26820 /$ recimundo/3.(3).septiembre.2019.1061-1084

URL: http://recimundo.com/index.php/es/article/view/559

Código UNESCO: 6114.12 Liderazgo

Tipo de Investigación: Artículo de Revisión

Editorial Saberes del Conocimiento

Recibido: 15/05/2019

Aceptado: 23/06/2019

Publicado: 30/09/2019

Correspondencia: fadnamay@hotmail.com

a. Magister en Administración de Empresas; Ingeniero Agropecuario con Mención en Gestión Empresarial Agropecuaria; Agrozootecnista; Universidad Técnica de Babahoyo; Babahoyo, Ecuador; fadnamay@ hotmail.com

b. Ingeniero Agrónomo; Magíster en Ingeniería Agrícola; Magister Ejecutivo en Dirección de Empresas con Énfasis en Gerencia Estratégica; Especialista en Gerencia de Proyectos; Universidad Técnica de Babahoyo; Babahoyo, Ecuador; dmayorga@utb.edu.ec

c. Doctora en Educación; Magister en Dirección de Empresas con Énfasis en Gerencia Estratégica; Diploma Superior en Investigación de la Educación a Distancia; Economista; Licenciada en Ciencias Económicas; Instituto Superior Tecnológico Juan Bautista Aguirre; Daule, Ecuador; idacampimayorga@ hotmail.com

d. Magister Ejecutivo en Dirección de Empresas con Énfasis en Gerencia Estratégica; Diploma Superior en Investigación de la Educación a Distancia; Economista; Licenciado en Ciencias Económicas; Universidad Regional Autónoma de Los Andes; Ambato, Ecuador; deluccaec@yahoo.com 


\section{Liderazgo: una habilidad gerencial fundamental en el éxito de una empresa en el siglo XXI}

Vol. 3, núm. 3., (2019)

Félix Adolfo Navia Mayorga; David Mayorga Arias; Ida Ivete Campi Mayorga; Luis Alberto De Lucas Coloma

\section{RESUMEN}

Una empresa que tenga en su visión llegar al éxito, tiene un gran desafío pues necesita de la presencia de un líder que motive y arrastre a los subordinados. Se realiza una revisión donde se consultaron 10 referencias bibliográficas de prestigiosas revistas científicas con un grado alto de actualización con respecto al tema que nos permitió obtener las definiciones existentes en las literaturas, pero todas concuerdan en su esencia que es el proceso de dirigir las actividades laborales de los miembros de un grupo y de influir en ellas, También se exponen los estilos de liderazgo desde lo tradicional hasta lo actual; en este caso con la descripción de los líderes estratégicos y los sostenibles, para el logro de los objetivos de la empresa o compañía.

Palabras Claves: Habilidades gerenciales; Gerencia de empresas; Concepto de líder; Lideres del siglo XXI. 


\title{
Liderazgo: una habilidad gerencial fundamental en el éxito de una empresa en \\ el siglo XXI
}

Vol. 3, núm. 3., (2019)

Félix Adolfo Navia Mayorga; David Mayorga Arias; Ida Ivete Campi Mayorga; Luis Alberto De

Lucas Coloma

\begin{abstract}
A company that has in its vision to succeed, has a great challenge because it needs the presence of a leader that motivates and draws subordinates. A review is made where 10 bibliographic references of prestigious scientific journals were consulted with a high degree of updating regarding the topic that allowed us to obtain the existing definitions in the literature, but all agree in essence that it is the process of directing work activities of the members of a group and of influencing them, Leadership styles are also exposed from the traditional to the current; in this case with the description of the strategic and sustainable leaders, for the achievement of the objectives of the company or company.
\end{abstract}

Key Words: Management skills, Business management, Leader concept, Leaders of the $21 \mathrm{st}$ century. 


\section{Liderazgo: una habilidad gerencial fundamental en el éxito de una empresa en el siglo XXI}

Vol. 3, núm. 3., (2019)

Félix Adolfo Navia Mayorga; David Mayorga Arias; Ida Ivete Campi Mayorga; Luis Alberto De Lucas Coloma

\section{Introducción.}

Numerosos son los intentos que se han efectuado por descubrir las habilidades que debe poseer un ejecutivo para cumplir con las variadas funciones de su cargo. Estas descripciones presentan variabilidad en las habilidades incluidas; dependiendo de los intereses de estudio de los autores; el nivel jerárquico del personal en que se centra el estudio y la industria para la que se definen las categorías de habilidades. Además se describen las habilidades analíticas; habilidades interpersonales y habilidades emocionales; en esta oportunidad se enfatiza dentro de las interpersonales: el Liderazgo.

Una organización pretende tener una planeación adecuada; control y buen procedimiento de organización y no sobrevivir a la falta de un líder apropiado, por lo que es vital para la supervivencia de cualquier negocio, empresa u organización, la presencia de un liderazgo dinámico.

Un individuo quizás sea un gerente eficaz, justo y organizado, pero carente de las cualidades del líder para motivar; tal vez otras personas sean líderes eficaces; con habilidad para desplegar el entusiasmo y la devoción; pero carentes de posibilidades para canalizar la energía que desatan en otros.

Recompensar al personal por trabajos excepcionales es básico para mantenerlos motivados y que den lo mejor de sí. Es importante lograr que estas recompensas generen orgullo, entusiasmo, diversión y agradables reconocimientos.

El gran desafío de las organizaciones, especialmente del liderazgo; consiste en la optimización de la función laboral, esto es; integrar el ser y el hacer del hombre. 


\section{Liderazgo: una habilidad gerencial fundamental en el éxito de una empresa en el siglo XXI}

Vol. 3, núm. 3., (2019)

Félix Adolfo Navia Mayorga; David Mayorga Arias; Ida Ivete Campi Mayorga; Luis Alberto De

Lucas Coloma

Las empresas contemporáneas están en la necesidad de contar con personas que puedan construir visiones más humanistas para generar con mediatez cambios novedosos y responsables socialmente útiles; estas son las razones por lo que el liderazgo se está convirtiendo en una profesión.

El liderazgo hace falta en el mundo de los negocios del siglo XXI, velozmente cambiante y competitivo.

Definiciones de Líder y Liderazgo

Inminentemente son extensas las definiciones de liderazgo; entendido por tantos autores, como expertos, investigadores se han dedicado al tema; algunas de las más significativas reseñan en el concepto como, Cito:

- El proceso que implica ejercer influencia sobre las actividades de un grupo organizado en los esfuerzos que este realiza para definir y alcanzar objetivos.

- La influencia interpersonal ejercida en una situación dirigida a través del proceso de comunicación humana a la consecución de uno o diversos objetivos específicos.

- El proceso de dirigir las actividades laborales de los miembros del grupo e influir en ellos.

- Es un proceso permanente de readecuación e inherente a toda la vida; ya que busca transformar, a todo momento las potencialidades de sus colaboradores; desarrollando sus capacidades, motivaciones y valores con vista a mejorar su desempeño laboral en el futuro. 


\section{Liderazgo: una habilidad gerencial fundamental en el éxito de una empresa en el siglo XXI}

Vol. 3, núm. 3., (2019)

Félix Adolfo Navia Mayorga; David Mayorga Arias; Ida Ivete Campi Mayorga; Luis Alberto De Lucas Coloma

- La capacidad de influir en la conducta de los demás; potenciando los objetivos particulares de las personas que colaboran con la organización y los del grupo en su conjunto

- Es un proceso de influencia sobre las actividades de individuos o grupos; para lograr metas comunes en situaciones determinadas.

- La influencia, arte o proceso de influir sobre las personas para que intenten con buena voluntad y entusiasmo el logro de las metas de la organización. (Figueroa Soledispa \& Machado Ramírez, 2012), (Hernández, 2014)

En esencia todas concuerdan, pudiéndose resumir como; el proceso de dirigir las actividades laborales de los miembros de un grupo; a través de la comunicación, y de la influencia en ellas; para el logro de los objetivos de la empresa o compañía. Es una habilidad que abarca múltiples cualidades personales de suma y relevante importancia para un gerente o directivo; pues se basa en la influencia interpersonal orientada hacia el logro de objetivos, a corto plazo (misión); o a largo plazo (visión); mediante la comunicación.

Ampliamente claro está, que un directivo que quiera ser líder; lo pueda lograr con la creación de actividades de capacitación; con el fin de desarrollar el liderazgo; como son el estudio de teorías, tácticas y estrategias de liderazgo; aunque asumir que con horas de capacitación sostendrán líderes, parece un poco irrisorio.

En Colombia constataron y declararon estudio realizado en bancarios, que; los líderes mantienen un mayor número de capacitaciones en el tema de liderazgo; aunque la participación de 


\section{Liderazgo: una habilidad gerencial fundamental en el éxito de una empresa en el siglo XXI}

Vol. 3, núm. 3., (2019)

Félix Adolfo Navia Mayorga; David Mayorga Arias; Ida Ivete Campi Mayorga; Luis Alberto De

Lucas Coloma

los seguidores o subordinados no se correspondan, de igual forma; aclarando que ha recibido capacitaciones en temas relacionados. (Pautt Torres \& Contreras M, 2012)

Siguiendo el camino del liderazgo, algunos expertos hacen alusión que existen otros factores que lo componen como son la genética e infancia; la educación; la experiencia; los fracasos y la formación objetiva. Para el desarrollo de esta habilidad es importante trabajar en la propia autoestima, expresado por (Huerta Mata y citado por Duarte Rodríguez): "La capacidad para desarrollar o fortalecer la confianza en uno mismo es la base para mejorar la habilidad del liderazgo. Si la gente considera que una persona confía en si misma, será relativamente fácil que la acepten como líder”. (Duarte Rodríguez, 2013)

En continuas y constante ocasiones se ha expresado que; ser líder, es un arte más que ciencia; que hay que saber conquistar con un aprendizaje continuo, en el cuál lo importante es no caer; sino levantarse siempre corrigiendo los errores que como seres humanos se pueden cometer. Lo importante es que quien se proponga formarse como líder empiece por reconocerse humildemente a sí mismo; un autoconocimiento sincero que le lleve a percibir sus inexactitudes y tomar un plan de acción concreto que permita corregirlas en forma y a tiempo. El líder debe aprender a valorarse con sus defectos y virtudes; que siempre que tome una decisión en cualquier ámbito de la empresa debe medir las consecuencias humanas o financieras que traerá dicha acción en el futuro posteriormente.

¿El líder nace o se hace?; es una pregunta frecuentemente utilizada en el campo de la administración; si queremos darle respuesta a esta pregunta no obviaremos que ciertas características innatas del individuo contribuyen de una manera importante a poseer la habilidad del 


\section{Liderazgo: una habilidad gerencial fundamental en el éxito de una empresa en el siglo XXI}

Vol. 3, núm. 3., (2019)

Félix Adolfo Navia Mayorga; David Mayorga Arias; Ida Ivete Campi Mayorga; Luis Alberto De Lucas Coloma

liderazgo de una manera natural; pero si afirmando que el entorno cambiante de la organización y las diferentes situaciones sociales requieren del aprendizaje y adaptación continua de esa habilidad; que se enriquece principalmente con el cúmulo de experiencias y la preparación técnica específica.

Fiestas Antón, 2008, en su ponencia sobre liderazgo plantea que, Cito: "La opinión generalizada es que hay líderes que nacen con capacidades innatas y hay otros que se van formando

en su desarrollo profesional."

Estas habilidades innatas favorecen el desarrollo del líder; pero a veces resulta más determinante la formación que uno va adquiriendo y la experiencia que va acumulando.

El liderazgo es considerado un fenómeno universal; tanto en el espacio como en el tiempo; apareciendo continuamente la figura del líder; la cual es una figura común a todas las comunidades humanas; en todas las etapas históricas por las que ha transitado la humanidad.

La dimensión de autoridad; se manifiesta de dos formas, según, (Álvarez 2001), se define liderazgo institucional; cuando la persona es elegida o nombrada por los miembros de la organización por su facultad de representar y dirigir formalmente a la institución; o como liderazgo profesional; ejercido por la persona que es elegida por los miembros de la institución; que demuestra mayores conocimientos, experiencia y capacidad para dirigir procesos; ya sea formal o informalmente; mientras que la dimensión de la influencia; es que el liderazgo es ejercido formal o informalmente; con una gran fuerza de atracción e implicación de sus seguidores, sin necesidad de imposición ni coacción. (Rosales, 2005) 


\section{Liderazgo: una habilidad gerencial fundamental en el éxito de una empresa en el siglo XXI}

Vol. 3, núm. 3., (2019)

Félix Adolfo Navia Mayorga; David Mayorga Arias; Ida Ivete Campi Mayorga; Luis Alberto De

Lucas Coloma

\section{Estilos tradicionales de liderazgo}

Si la forma en cómo los líderes dirigen a sus seguidores se puede llegar a una clasificación; no existe un estilo mejor o peor que otro, sino que depende del grado de adecuación a la situación del grupo; la cultura organizacional; características de los seguidores; además de la relación que el líder establece con ellos, por otro lado, algunos expertos en desarrollo organizacional, hacen el planteamiento; que existen múltiples tipos de liderazgo; asumiendo otros que el liderazgo es uno y, como los líderes son individuos con características personales definidas; las clasificaciones corresponden a la forma en cómo ejercen o han adquirido la facultad de dirigir.

Lussier y Achua, a finales de los años 40, expertos en el tema de liderazgo se enfocaron en la teoría del comportamiento y partiendo de la autoridad del líder describieron los estilos de liderazgo.

Un primer planteamiento (Kurt Lewin y colaboradores), identifican dos estilos de liderazgo; autocrático y democrático, posteriormente Koontz y Weihrich; agregan a los anteriores el estilo de liderazgo liberal. Es importante tener en cuenta que, de acuerdo a las características actuales de la sociedad en la que se promueven las relaciones dialógicas por sobre las hegemónicas y la participación por sobre la pasividad; se privilegian aquellos estilos que facilitan una mayor participación del grupo, en la toma de decisiones de la organización.

Al tratarse de la dimensión poder: Una vez que este ha sido alcanzado; se habla del líder Carismático; elegido como líder por su manera de entrega que sus seguidores; es el que tiene la capacidad de generar entusiasmo. Tradicional; es aquel que hereda el poder por costumbre o por un 


\section{Liderazgo: una habilidad gerencial fundamental en el éxito de una empresa en el siglo XXI}

Vol. 3, núm. 3., (2019)

Félix Adolfo Navia Mayorga; David Mayorga Arias; Ida Ivete Campi Mayorga; Luis Alberto De Lucas Coloma

cargo importante. Legítimo; es el que adquiere el poder mediante procedimientos autorizados en las normas legales de la institución o empresa.

Otros autores (Vargas), expone la clasificación de: Autocrático; cuando el poder está centrado y la toma de decisiones monopolizada; los empleados se limitan a cumplir órdenes temiendo a las amenazas y castigos; este liderazgo autocrático se torna negativo debido al temor generado y a la frustración prevalente, ya que los gerentes de estas organizaciones son verdaderos dictadores; por lo tanto que el grupo es ignorado. Participativo; los integrantes de la empresa actúan corno una unidad con el gerente a la cabeza; como orientador y guía; las decisiones florecen de la consulta con la debida participación de los seguidores. Anárquico; las organizaciones sobresalen por la anarquía reinante; el gerente elude su poder y su responsabilidad; solo la automotivación de los integrantes del grupo mantiene la actividad; el líder es ignorado por el grupo.

Inferimos que (Molinar y Velázquez, 2005); hacen la proposición de un resumen de la propuesta de (Levicki 2000): que descarta la distinción entre los diferentes estilos de liderazgo: Liderazgo autocrático: Centrado en sí mismo; dominante; dirige desde el centro; envía las respuestas desde su cargo; no consulta; el líder define los roles y les orienta a las personas qué hacer, cómo y cuándo. Líder tolerante: Deja que las personas resuelvan los conflictos; casi nunca se involucra y participa poco; su función principal es facilitar y comunicar. Líder democrático: Es participativo; conoce la respuesta; irradia que las personas se sientan confiadas en su tarea y dignas de confianza; consulta a los demás las decisiones, manifiesta un comportamiento directivo y de apoyo a la vez. Líder paternalista: El mismo resuelve los problemas de los demás; casi no permite la consulta y brinda poca dirección y apoyo. (Noriega Gómez, 2008) 


\section{Liderazgo: una habilidad gerencial fundamental en el éxito de una empresa en el siglo XXI}

Vol. 3, núm. 3., (2019)

Félix Adolfo Navia Mayorga; David Mayorga Arias; Ida Ivete Campi Mayorga; Luis Alberto De

Lucas Coloma

Características generales del líder

La concreta y evidente definición de liderazgo enumera ya varias características del líder, a continuación se describen las siguientes:

- Capacidad de comunicarse. La comunicación es en dos sentidos; debe expresar claramente sus ideas y sus instrucciones; lograr que su gente las escuche y las entienda; debe saber escuchar y considerar lo que el grupo al que dirige le expresa.

- Inteligencia emocional. Estudiosos del tema definieron inicialmente la Inteligencia Emocional como, Cito: la habilidad para manejar los sentimientos y emociones propios y de los demás, de discriminar entre ellos y utilizar esta información para guiar el pensamiento y la acción. Los sentimientos mueven a la gente, sin inteligencia emocional no se puede ser líder.

- Capacidad de establecer metas y objetivos. Para dirigir un grupo, hay que saber a donde llevarlo; sin una meta clara, ningún esfuerzo será suficiente; las metas deben ser congruentes y certeras con las capacidades del grupo; de nada sirve establecer objetivos que no se pueden cumplir.

- Capacidad de planeación. Establecida la meta; es necesario ejecutar un plan para llegar a ella; en este plan se deben definir las acciones que deben cumplirse; el momento en que se deben realizar; las personas encargadas de ellas, así como los recursos necesarios, etc. Un líder reconoce sus fortalezas y las aprovecha al máximo; por supuesto también debe saber cuáles son sus debilidades y buscar subsanarlas. 


\section{Liderazgo: una habilidad gerencial fundamental en el éxito de una empresa en el siglo XXI}

Vol. 3, núm. 3., (2019)

Félix Adolfo Navia Mayorga; David Mayorga Arias; Ida Ivete Campi Mayorga; Luis Alberto De Lucas Coloma

Un líder crece y hace crecer a su gente; para ese crecimiento no se aferra a su puesto y actividades actuales; siempre ve hacia arriba, para crecer enseña a su gente delegando funciones además de crear oportunidades para todos.

Tiene carisma. Carisma es el don de atraer y caer bien, llamar la atención y ser agradable a los ojos y percepción de las personas; para adquirir ésta característica basta con interesarse por la gente y demostrar verdadero interés en ella; la realidad, es que en el carisma prima la excelencia, se nutre con excelencia; porque es lo más alejado que hay del egoísmo; cuando un líder pone toda su atención en practicar los hábitos de la excelencia, el carisma llega y como una avalancha cae un torrente de agradables ideas sobre el líder.

Es Innovador. Este siempre está en busca de nuevas y mejores maneras de hacer las cosas. Esta característica es importante ante un mundo que avanza rápidamente, con tecnología cambiante y ampliamente competitivo.

Un líder es responsable; Sabe que su liderazgo le ofrece poder, utilizándolo en beneficio de todos.

Un líder está informado. Se hace evidente que ninguna organización puede sobrevivir sin líderes que entiendan o sepan cómo se maneja la información; un líder debe saber cómo se procesa la información, interpretarla inteligentemente y utilizarla en la forma más moderna, creativa y adecuada. 


\section{Liderazgo: una habilidad gerencial fundamental en el éxito de una empresa en el siglo XXI}

Vol. 3, núm. 3., (2019)

Félix Adolfo Navia Mayorga; David Mayorga Arias; Ida Ivete Campi Mayorga; Luis Alberto De

Lucas Coloma

Características de los líderes exitosos

Los individuos que logran éxito al frente de las empresas que dirigen, muestran ciertos rasgos que los diferencian de los demás; estas se caracterizan por ser;: Emprendedoras, enérgicas y ambiciosas; gustan de los retos, proponiéndose tareas difíciles y queriendo ser siempre los primeros; tenaces ya que no desmayan en sus intentos; convierten sus problemas en retos y disfrutan del triunfo.

Honestos y Confiados. Recompensan a sus colaboradores de forma justa; confían en la capacidad, inteligencia y lealtad del grupo que los rodea sabiendo delegar responsabilidades.

Seguros de su capacidad. Se comprometen porque tienen seguridad en el cumplimiento de las metas; se reconocen a sí mismos y por lo tanto saben de qué son capaces.

Flexibles en sus estrategias. Entienden que la administración es contingencial y por lo tanto hay un tratamiento especial para cada situación; sus estrategias flexibles le permiten opciones múltiples en la solución de problemas.

Creativos. Esto les permite ser activos en lugar de reactivos utilizando la mejor manera de hacer las cosas y saber comunicar al grupo su visión.

Visionarios. Proveen el futuro; son perspectivos y se convierten en agentes del cambio, antes de ser víctimas de él.

Motivadores. Crean, con su ejemplo el ambiente propicio y motivan a sus colaboradores para lograr su aporte en pro de los objetivos trazados. 


\section{Liderazgo: una habilidad gerencial fundamental en el éxito de una empresa en el siglo XXI}

Vol. 3, núm. 3., (2019)

Félix Adolfo Navia Mayorga; David Mayorga Arias; Ida Ivete Campi Mayorga; Luis Alberto De Lucas Coloma

Los hombres de éxito son entusiastas; positivos y no se desaniman ante los pesimistas; huyen de lo negativo y creen en el futuro.

- Líder empresarial. Como verdadero líder; el empresario es emprendedor; ambicioso; tenaz; es una persona de visión que descubre las oportunidades y emprende la acción, su emotividad y seguridad en sí mismo le permite incrementar el número de seguidores; es Quien hace empresa; persona positiva; amante del riesgo: con seguridad del éxito, los fracasos son simples experiencias y los éxitos, pese a que los disfrutan, son tomados como algo natural. Y seguro; como buen Líder el empresario siempre se atreve y trata de rodearse solo de personas positivas, difícilmente aceptan un No.

Cita: un ejemplo de líder empresarial:

"En 1946 Estados Unidos carecía de un servicio de alquiler de carros en los aeropuertos, pues la agencia Hertz no prestaba este servicio en los terminales aéreas. Los ejecutivos que viajaban no tenían la oportunidad de utilizar estos servicios; además, no había tarjetas de crédito para tal fin.

Warren Avis prestaba el servicio militar y en consecuencia viajaba constantemente, sintiendo la necesidad de este servicio. Esto lo llevó a plantear la idea de crear un servicio de alquiler de coches en los aeropuertos, pero las personas a quienes les propuso la idea no la vieron viable porque consideraban que la demanda a nivel nacional era muy baja y los costos altos, descartando cualquier apoyo a la misma.

Avis no se dejó desanimar por los pesimistas y con 10.000 dólares propios y un préstamo de 75.000 dólares, avalado mediante pagaré personal, a finales de la década del 40 AVIS-RENT-A- 


\section{Liderazgo: una habilidad gerencial fundamental en el éxito de una empresa en el siglo XXI}

Vol. 3, núm. 3., (2019)

Félix Adolfo Navia Mayorga; David Mayorga Arias; Ida Ivete Campi Mayorga; Luis Alberto De

Lucas Coloma

CAR, empezó a funcionar en Detroit y Miami, por ser los aeropuertos con mayor movimiento. Los mostradores donde se anunciaba el servicio fueron colocados estratégicamente cerca de donde se entregaban los equipajes y allí se le explicaba a la gente, aprovechando la demora en la entrega de las maletas.

Después el servicio se extendió a Nueva York, Chicago, Dallas y Texas, con contratos de exclusividad firmados con las administraciones respectivas. En 1957 Avis ocupó el segundo lugar a nivel mundial, detrás de la Hertz; pero la AVIS era la única que prestaba este servicio en los aeropuertos con una tarjeta de crédito exclusiva para tal fin.

AVIS convenció a los directivos de las principales líneas aéreas de las ventajas que representaban para unos y otros hacer alianzas estratégicas para defender intereses comunes. Fue así como en los principales periódicos empezaron a salir anuncios conjuntos, se colocaban folletos de la AVIS en los bolsillos de las sillas de los aviones y las empresas de aviación accedieron a prestar los teletipos en forma gratuita para reservación de coches". (Vargas Tolosa, 2012)

\section{Lideres y Gerentes}

Actualmente existen confusiones que se han generado entre estos dos términos; liderazgo y dirección, ya que frecuentemente son utilizados dentro del lenguaje administrativo; como si fueran iguales; por tales razones se establece una diferencia entre lo que hace un líder dentro de la empresa y con su equipo de trabajo y qué logros obtiene; y lo que hace un directivo dentro de la empresa y con su equipo de trabajo y los logros que obtiene. Estos dos términos se diferencian por sus actitudes, métodos y motivaciones; mientras que los gerentes crean estructuras corporativas 


\section{Liderazgo: una habilidad gerencial fundamental en el éxito de una empresa en el siglo XXI}

Vol. 3, núm. 3., (2019)

Félix Adolfo Navia Mayorga; David Mayorga Arias; Ida Ivete Campi Mayorga; Luis Alberto De Lucas Coloma

adecuadas y se sienten distanciados emocionalmente de su trabajo; los líderes se mantienen en la búsqueda de introducir nuevos enfoques e ideas en la organización, ya que están comprometidos con ella. (Pautt Torres, 2011)

Los Gerentes y los Líderes son individuos típicos muy distintos de personas en su manera de pensar y de actuar frente a si mismos como frente a los demás; los primeros tienden a adoptar actitudes interpersonales a las metas, las cuales nacen de necesidades más que de deseos; mientras los segundos, como verdaderos transformadores; se comprometen y comprometen a los demás con la organización en la búsqueda de los objetivos. Los verdaderos líderes solo estarán satisfechos cuando logren sus resultados deseados.

La línea divisoria entre liderazgo y gerencia; en el ansia de ser explicado por algunos autores, ha resultado siempre confusa y sutil; estos diferencian a cada uno de los términos expresando que los gerentes son personas que hacen el derecho de las cosas y los líderes son personas que hacen las cosas al derecho; pero no cabe la duda que existen diferencias notables entre el líder y el gerente o directivo, referidas como; los líderes son activos y como tales son creativos, pues le dan forma a las ideas; predicen el mundo cambiante y trazan los objetivos de la empresa, los gerentes, en cambio son reactivos, responden a las ideas y antes de crear hacen en la mayoría de las veces lo que hacían sus predecesores.

- En cuanto a las competencias entre uno y otro, se especifican que el líder inspira y compromete a su equipo para el logro de una visión, mientras que el directivo, gestiona; cumple con la misión de la organización y consigue la obediencia del grupo. 


\section{Liderazgo: una habilidad gerencial fundamental en el éxito de una empresa en \\ el siglo XXI}

Vol. 3, núm. 3., (2019)

Félix Adolfo Navia Mayorga; David Mayorga Arias; Ida Ivete Campi Mayorga; Luis Alberto De

Lucas Coloma

- En los gerentes existe la preocupación cómo hacer las cosas; mientras que los líderes se preocupan por la significación que tienen las cosas para las personas.

- El objetivo primordial del liderazgo es centrarse en los resultados, especialmente en los de tipo no cuantitativo; mientras que el propósito de la dirección o gerencia es mantener funcionando el sistema global de la organización, aunque a expensas del bienestar de los empleados.

- Un gerente asegura que se realice el trabajo y un líder se centra en la gente que hace el trabajo.

Los gerentes más eficaces se convierten en líderes y para llegar a ello, se han identificado dos factores críticos para el éxito; el aprendizaje y la participación del directivo.

\section{Liderazgo directivo}

Teniéndose en cuenta este tema tan controvertido, asimismo describir el considerado termino liderazgo -directivo; sumatoria de lo que es y hace el directivo más lo que es y hace el líder; para encontrar balanza, entre ambos requiere de gestión, resultados de inspirar, generar compromiso y confianza. Si se alcanza el equilibrio la empresa alcanzará los mejores resultados organizacionales con un capital humano comprometido y satisfecho, pero con cuidado porque puede inclinarse hacia un lado o el otro; si se inclina hacia el lado derecho, los resultados serían distintos a si ocurre lo contrario (Figura) 


\section{Liderazgo: una habilidad gerencial fundamental en el éxito de una empresa en el siglo XXI}

Vol. 3, núm. 3., (2019)

Félix Adolfo Navia Mayorga; David Mayorga Arias; Ida Ivete Campi Mayorga; Luis Alberto De Lucas Coloma

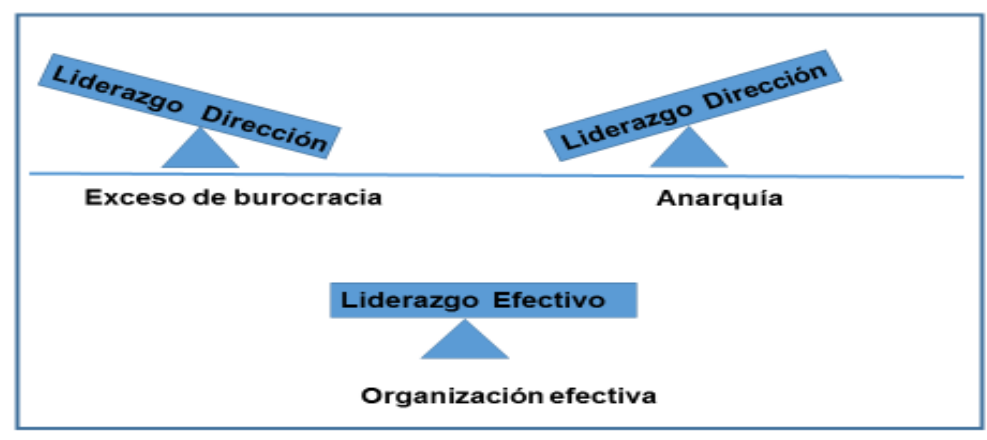

Figura 1. Balanza del Liderazgo Directivo. Tomado de Geili Pautt Torres

Se ha arribado al siglo XXI y las condiciones actuales presentes difieren de los siglos anteriores lo cual constituye un reto para las administraciones actuales el logro de objetivos de sustentabilidad y competitividad de las empresas; es así como se proponen dos nuevos tipos de líderes: el estratégico y el sostenible o sustentable, (algunos autores lo denominan Liderazgo en la "Nueva Edad"). Hagamos entonces una breve incursión para su interpretación:

\section{Liderazgo estratégico}

En época tan cercana como el 2011, queda definido que el Liderazgo Estratégico; como la capacidad de anticipar, vislumbrar y mantener la flexibilidad en la administración total de la organización a través de las personas, la delegación de facultades para crear y afrontar el cambio estratégico, cuando sea preciso, (planteado por Chiavenato y Sapiro ), Cito: 


\section{Liderazgo: una habilidad gerencial fundamental en el éxito de una empresa en el siglo XXI}

Vol. 3, núm. 3., (2019)

Félix Adolfo Navia Mayorga; David Mayorga Arias; Ida Ivete Campi Mayorga; Luis Alberto De

Lucas Coloma

Los rasgos que deben caracterizar a los líderes estratégicos en la actualidad para su contribución a mejorar el desempeño de sus recursos humanos; siendo este el objetivo del Modelo Estratégico Moderno de liderazgo (MEM) que satisface los requisitos antes planteados, con base en la literatura sobre las características del liderazgo moderno y su relación con el capital humano, así como en el análisis de cada uno de los catorce puntos que conforman éste modelo propuesto (Vargas y Guillén en el 2005); según estos autores, Cito: "el liderazgo estratégico influye en las personas en tres niveles: en la estructura sistémica (generativo), en los patrones de comportamiento (responsivo) y en los procesos y eventos (reactivo)." Otro autor lo define partiendo de su estrecha relación con el capital humano, Cito: "su logro se alcanza mediante la motivación, el compromiso y el desarrollo integral de las facultades humanas de sus seguidores"

Sabido es por todos, que en una organización la calidad del recurso humano es considerada el factor más importante que determina el nivel de éxito o fracaso de cada institución logrando mantener la competitividad estratégica.

Se describe por estudiosos del tema, la propuesta de las 14 características del modelo de liderazgo estratégico moderno (MEM); las que por la amplia extensión de la propuesta no se hace posible profundizar en cada una de ellas; pero si hacemos mención de las mismas, que son: Prácticas visionarias, Orientado al proceso y al prospecto (Cliente), Confidente pero sin arrogancia, Busca adquirir y capitalizar los conocimientos, Busca liberar y nutrir la creatividad de las personas, Flujos de trabajo influenciados por las relaciones, Determina la importancia de la integridad por las acciones, Voluntad para ganar respeto, Busca la diversidad, Actúa para anticipar el cambio ambiental, Sirve como líder y como un gran miembro del grupo, Ve a los colaboradores de la 


\section{Liderazgo: una habilidad gerencial fundamental en el éxito de una empresa en el siglo XXI}

Vol. 3, núm. 3., (2019)

Félix Adolfo Navia Mayorga; David Mayorga Arias; Ida Ivete Campi Mayorga; Luis Alberto De Lucas Coloma

organización como un recurso crítico, Opera a través de un estado mental global, Invierte en la continuidad del desarrollo de colaboradores. (Lezama Torres, Cruz Vásquez, \& Pico González)

\section{Liderazgo sostenible o sustentable}

Aún es un tema poco explorado, así su importancia como estrategia en las organizaciones. Infiriéndose que la imagen de un líder sostenible en el mundo global de los negocios; hace necesario que incorpore estrategias de responsabilidad social y de sustentabilidad en sus organizaciones; la sustentabilidad basada en los aspectos económicos, ambientales y sociales; otros añaden, en lo familiar, la cultura, las guerras, lo tecnológico o lo político, deben incluirse en las estrategias empresariales.

Observable es que este nuevo modelo de liderazgo se significa como parte de una educación basada en lo colectivo y en la conciencia global acerca del medio ambiente y de las cuestiones sociales; El papel social se convierte en una característica diferencial competitiva en los negocios y las empresas que descubren la importancia de la gestión ética de sus marcas y productos. Los buenos gestores son importantes para cualquier empresa y cualquier mercado, pero los líderes con principios y prácticas sostenibles son imprescindibles; la incorporación de la sustentabilidad y de la responsabilidad social como valores de la empresa es una práctica que el líder sostenible debe perseguir; ya que es fundamental que existan en la avanzada de las organizaciones líderes que hayan incorporado la cultura de la sustentabilidad. 


\section{Liderazgo: una habilidad gerencial fundamental en el éxito de una empresa en el siglo XXI}

Vol. 3, núm. 3., (2019)

Félix Adolfo Navia Mayorga; David Mayorga Arias; Ida Ivete Campi Mayorga; Luis Alberto De

Lucas Coloma

El asunto económico prevalece hoy día en la formación de gestores; siendo este un este compromiso económico, social y medioambiental lo que marcará la diferencia para las generaciones futuras.

El líder sostenible; promulgado como un agente de cambio en dirección hacia la sustentabilidad pues el rumbo que tomará el planeta en las próximas décadas aun es incierto, teniéndose en cuenta que las Naciones Unidas viene declarando informes sobre el Medio ambiente en diferentes Cumbres o Conferencias Mundiales, desde 1972 al 2015. Recordar que, en 1982, surge el término de "desarrollo sostenible o sustentable" a partir del informe de la problemática ambiental universal y su grado de deterioro, entre ellas la pérdida anual de los 150000 kilómetros cuadrados de bosque tropical; el deterioro de 6 a 10 millones de hectáreas de suelo agrícola por erosión, salinidad y otros procesos, la escasez y mala utilización del agua, la emisión a la atmosfera de millones de toneladas de gases contaminantes, sequías e inundaciones provocadas por el cambio climático y otros riesgos inminentes palpables en todo el mundo. En 1992 se celebró la famosa Cumbre de la Tierra en Rio de Janeiro con el fin de firmar Acuerdos globales (Agenda 21), ya que la situación planteada 10 años atrás, ni 20 años después (año 2015) se había revertido, firmándose entonces la "Agenda 2030 para el desarrollo sostenible".

Este recuento muestra que las perspectivas sobre el camino hacia la sustentabilidad van a estar en constante riesgo, siendo necesario desarrollar a los líderes sostenibles o sustentables para que desplieguen una concientización adecuada sobre los aspectos medioambientales, así como una responsabilidad social traducida en la adopción de prácticas que van más allá de los deberes básicos; por lo que es inminente la exigencia de un compromiso mayor de éstos líderes; es común que las 


\section{Liderazgo: una habilidad gerencial fundamental en el éxito de una empresa en el siglo XXI}

Vol. 3, núm. 3., (2019)

Félix Adolfo Navia Mayorga; David Mayorga Arias; Ida Ivete Campi Mayorga; Luis Alberto De Lucas Coloma

empresas solamente cumplan con lo que se impone por ley; pero el liderazgo sostenible puede ofrecer más al aplicar conceptos de sustentabilidad y responsabilidad social.

Existen algunos atributos de este nuevo líder, que se añaden a los tradicionales ya referidos, entre ellos están:

- Difundir los conceptos de sustentabilidad y responsabilidad social interna y externamente (stakeholders)

- Mantener el equilibrio de los aspectos económicos, sociales y medioambientales de la empresa

- Liderar la elaboración de una estrategia consistente de sustentabilidad para la empresa

- Revisar los productos y procesos que puedan provocar impactos medioambientales negativos

- Coordinar el proceso de cambio de mentalidad con la introducción de las dimensiones económicas, sociales y medioambientales en la noción de éxito empresarial estableciendo una visión y una misión de sustentabilidad.

Inspirar a los seguidores con relación a las causas de la sustentabilidad y no imponerlas mediante la autoridad. (Mello, 2015)

\section{Consideraciones finales}

Son extensas las conceptualizaciones del liderazgo tradicional descrito desde tiempos inmemorables por la Ciencia de la Administración y todos los estudiosos del tema están de acuerdo en que el líder es ÊL que arrastra y motiva a las personas a trabajar en un ambiente adecuado que permite el aumento de la productividad de la empresa o calidad de los servicios que se brinda; se 


\section{Liderazgo: una habilidad gerencial fundamental en el éxito de una empresa en \\ el siglo XXI}

Vol. 3, núm. 3., (2019)

Félix Adolfo Navia Mayorga; David Mayorga Arias; Ida Ivete Campi Mayorga; Luis Alberto De

Lucas Coloma

hace necesario en estos tiempos desarrollar la actuación del liderazgo estratégico y el liderazgo sostenible como nuevo modelo del liderazgo moderno en las empresas del siglo XXI; ya que resulta vital para las empresas y son estos líderes los encargados de coordinar el proceso de cambio de mentalidad hacia la sustentabilidad; cuyos aspectos buscan el desarrollo equilibrado.

Los líderes necesitan saber cómo se utilizan las nuevas tecnologías; van a necesitar saber cómo pensar para poder analizar y sintetizar eficazmente la información que están recibiendo a pesar de las nuevas tecnologías; su dedicación debe seguir enfocada en el individuo. Sabrán que los líderes dirigen gente: no cosas; números o proyectos. Tendrán que ser capaces de suministrar lo que la gente quiera con el fin de motivar a quienes están dirigiendo. Tendrán que desarrollar su capacidad de escuchar para describir lo que la gente desea, per también tendrán que desarrollar su capacidad de proyectar, tanto a corto como a largo plazo, para conservar un margen de competencia.

\section{Bibliografía.}

Duarte Rodríguez, A. C. (2013). Habilidades gerenciales y su importancia para el exito de una organización. Bogota: Universidad Militar Nueva Granada. Recuperado el 5 de septiembre de 2018

Figueroa Soledispa, M. L., \& Machado Ramírez, E. F. (2012). La superación en liderazgo docente y la tranformación de los procesos universitarios. Humanidades Médicas, 12(3), 391-408. Recuperado el 13 de septiembre de 2018, de Humanidades Médicas .

Hernández, A. P. (2014). Influencia del liderazgo sobre el clima organizacional. Suma de Negocios, 5(11), 117-125. Recuperado el 10 de septiembre de 2018

Lezama Torres, M. A., Cruz Vásquez, M., \& Pico González, B. (s.f.). El liderazgo estratégico modeno y el mejoramiento del desempeño del capital humano. Recuperado el 9 de septiembre de 2018, de Red Internacional de Investgafores en Competitividad .Memoria del IX Congreso: https://www.riico.net/index.php/riico/article/viewFile/15/15 


\section{Liderazgo: una habilidad gerencial fundamental en el éxito de una empresa en el siglo XXI}

Vol. 3, núm. 3., (2019)

Félix Adolfo Navia Mayorga; David Mayorga Arias; Ida Ivete Campi Mayorga; Luis Alberto De Lucas Coloma

Mello, M. F. (2015). La importancia del liderazgo sostenible como una estrategia de las organizaciones. Recuperado el 2 de septembre de 2018, de Revista de Ciencias Estratégicas

23(34),209-218: https://revistas.upb.edu.co/index.php/cienciasestrategicas/article/view/7369

Noriega Gómez, M. G. (2008). La importancia del liderazgo en la organizaciones . Recuperado el 12 de septiembre de 2018, de Tema de Ciencias y Tecnología.12(36),25-29: http://www.utm.mx/edi_anteriores/temas036/ENSAYO3-36.pdf

Pautt Torres, G., \& Contreras M, C. A. (2012). Factores que influyen en el liderazgo de los gerentes del sector bancario en la ciudad de Cúcuta-Norte de Santander. Revista Escuela de Administración de Negocios, 73(1), 80-95. Recuperado el 22 de agosto de 2018

Rosales, C. (02 de 05 de 2005). La actitud del líder. Recuperado el 10 de septiembre de 2018, de https://degerencia.com/articulo/la_actitud_del_lider/

Vargas Tolosa, J. R. (2012). El liderazgo .Un enfoque moderno. Recuperado el 11 de septiembre de 2018, de Editorial Vamel Vargas Melendez 2da Edición .Cúrcuta.Colombia : http://roa.ult.edu.cu/.../LIDERAZGO\%20MODERNO\%20(LIBRO).doc 